\title{
Excitation Wavelength and Temperature at Irradiation of Metal by Atmospheric- Pressure Nonequilibrium Argon Plasma
}

\author{
Kiyoyuki Yambe* \& Seiya Abe \\ Graduate School of Science and Technology, Niigata University, Niigata 950-2181, Japan \\ *Address all correspondence to: Kiyoyuki Yambe, Graduate School of Science and Technology, Niigata University, \\ Niigata 950-2181, Japan; Tel.: +81-25-262-6140; Fax: +81-25-262-6730, E-mail: yambe@eng.niigata-u.ac.jp
}

\begin{abstract}
We studied excitation wavelength and temperature during irradiation of atmospheric-pressure nonequilibrium argon plasma to pure metal plate. We observed excitation emission of light activated by argon plasma in the same wavelength region, both with and without metal plate. The wavelength region activated by atmospheric-pressure nonequilibrium argon plasma was limited. In the presence of the ion corresponding to wavelength region activated by argon plasma, corresponding ions were activated. Both oxygen ions and metal ions are generated by plasma irradiation, but during each observed wavelength, the wavelength of metal ion activated by argon plasma was dominant, because excitation energy of oxygen ion is greater than that of each metal ion. Heat propagation from argon plasma to metal plate occurs by plasma irradiation. Consequently, heat propagation from argon plasma to copper plate is greater in comparison to cases with other metal plates. This is due to the high thermal conductivity of copper. In the case of atmospheric-pressure nonequilibrium plasma, plasma is affected by interaction with an object, because plasma energy is smaller, and temperature of nonequilibrium plasma changes via with thermal conductivity of the object.
\end{abstract}

KEY WORDS: atmospheric-pressure nonequilibrium plasma, irradiation of metal, excitation wavelength, excitation temperature, heat propagation

\section{INTRODUCTION}

Recently, atmospheric-pressure nonequilibrium plasma techniques have been adopted for industrial, biological, and medical applications. ${ }^{1,2}$ Atmospheric-pressure nonequilibrium (cold) plasma formed by dielectric barrier discharge is intermittently generated using a dielectric, rare gas, and metal electrode by applying high-voltage alternating current (AC). ${ }^{3,4}$ Reports have described the properties of this type of plasma that is formed using helium or argon. ${ }^{5,6} \mathrm{~A}$ quartz tube is used as the dielectric, and the plasma is released into the atmosphere. A plasma jet has a small bullet-like volume that travels at unusually high velocity, ${ }^{7-10}$ but the estimated drift velocity of its ions is almost zero. ${ }^{11}$ The plasma jet is seen as the continuous movement of the plasma bullet. The travel length of the plasma jet into the atmosphere is influenced by the gas-flow state $12-16$ and varies with strength, duration, rise time, and polarity of the applied voltage. ${ }^{17-19}$ Consequently, the travel length of the plasma depends on plasma jet charge that changes with both electric field and resistive force caused by collisions between plasma and air. ${ }^{20-23}$ Plasma parameters (density and temperature) change with operation conditions, 
but with typical parameters, atmospheric-pressure nonequilibrium (cold) plasma can have a wide-ranging density of $10^{17}-10^{20} \mathrm{~m}^{-3}$, and its temperature can be a few electron volts. ${ }^{5,6,19,24-26}$

Plasma intermittently generated by AC power supply is in a nonthermal equilibrium state. One study reported such plasma's electron temperature to be $\sim 1 \mathrm{eV},{ }^{26}$ but ion and gas temperatures were close to room temperature. ${ }^{11}$ Therefore, it is thought that thermal damage to an object does not occur with plasma irradiation. However, a burn can occur to a human body with plasma irradiation, because the temperature at plasma jet contact has been measured to be $\sim 500 \mathrm{~K}$ with the use of a thermocouple under specific irradiation conditions. Plasma temperature may also be investigated using a spectrometer. When the temperature of plasma follows a Maxwell-Boltzmann distribution, this characteristic appears on the emission spectrum. ${ }^{27}$ When plasma is irradiated to a metal, the energy distribution of an ion activated by the plasma on the surface of the metal should show a Maxwell-Boltzmann distribution, because plasma causes heat propagation to an object. To safely use plasma irradiation and mechanism elucidation of the phenomenon to occur by plasma irradiation, it is important to clarify the characteristics of heat propagation from plasma to object.

In this work, we generated atmospheric-pressure nonequilibrium plasma by applying high-voltage AC using a quartz tube, argon gas, and copper foil electrode. We studied the excitation temperature of the argon plasma, oxygen ion, and metal ion, which we estimated using a line-pair method. ${ }^{27}$ Heat propagation from plasma jet to metal plate was investigated using excitation temperature after irradiation of each metal plate.

\section{EXPERIMENTALSETUP}

Figure 1 shows a schematic diagram of the irradiation of metal plate by an atmosphericpressure nonequilibrium plasma device, composed of a quartz tube and copper electrode, and its associated measurement system. The quartz dielectric tube had an inner diameter of $\sim 1.5 \mathrm{~mm}$ and a thickness of $\sim 1 \mathrm{~mm}$. The powered electrode was comprised of $20 \times 20$ $\mathrm{mm}$ copper foils with thickness of $0.1 \mathrm{~mm}$. The distance between the powered electrode and the edge of the quartz tube was $30 \mathrm{~mm}$. The gas-flow rate of argon gas was maintained at 0.8 standard liters per minute using a gas mass flow controller. We used an AC power supply (LHV-13A, Logy Electric, Tokyo, Japan) that was able to produce voltage from -6 to $+9 \mathrm{kV}$ and a main frequency of $\sim 13 \mathrm{kHz}$; the usual waveform is an asymmetric sine wave about the ground line. ${ }^{22}$ Plasma emission light was measured using a spectrometer (StellarNet, Inc. BLUE-Wave; Tampa, FL) through an optical fiber. The spectrometer used an exposure time of $100 \mathrm{~ms}$. The measurement point was set on the downstream side, and composition of the pure metal plates used for plasma irradiation was nickel (120.0 $\times 100.0 \times 0.1 \mathrm{~mm})$, copper $(60.0 \times 50.0 \times 0.3 \mathrm{~mm})$, and aluminum $(100.0 \times 100.0 \times 0.1$ $\mathrm{mm})$. The metal plate was set on a wood base at a distance of $10 \mathrm{~mm}$ from the edge of the quartz tube. Plasma was irradiated on the metal plate from above. The plasma jet touched the metal plate because plasma jet length was $\sim 40 \mathrm{~mm}$ from the edge of the quartz tube on the downstream side (in the case without metal plate). ${ }^{16}$ 


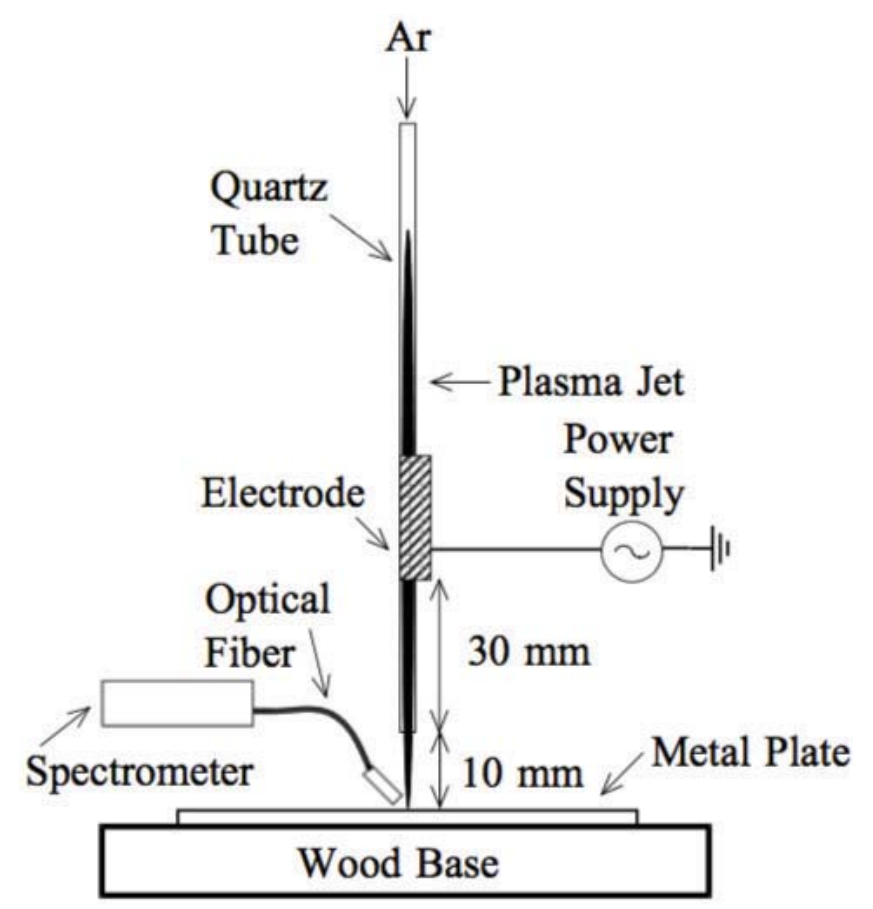

FIG. 1: Schematic diagram of the irradiation of metal plate by atmospheric-pressure nonequilibrium plasma device and its associated measurement system

\section{RESULTS AND DISCUSSION}

\section{A. Excitation Wavelength}

Figure 2 shows the spectral lines of the argon plasma, obtained at $15 \mathrm{~mm}$ from the edge of the powered electrode inside the quartz tube on the downstream side. The excitation wavelengths were observed to be between 700 and $920 \mathrm{~nm}$. Argon ion (Ar I) excitation appeared at wavelengths of 707, 738, 801, and $912 \mathrm{~nm}$ that were caused purely by the argon ion itself. Figure 3 shows the spectral lines of the argon plasma, oxygen ion, and copper ion obtained at the irradiation point of $10 \mathrm{~mm}$ from the edge of the quartz tube on the downstream side, in cases without and with copper plate. In the case without copper plate, the measurement point was on the surface of the wood base. In the case with copper plate, the measurement point was on the surface of the copper plate. The excitation wavelengths by plasma irradiation were observed to be between 300 and $410 \mathrm{~nm}$ outside of the quartz tube at the atmosphere region. The spectral-line intensities in the case with copper plate were greater than those without copper plate. The strong emission of light was also confirmed by viewing at a contact point of plasma jet and copper plate. In the case with metal plate, the electric field between the powered electrode and metal plate becomes strong with gathering of electric lines of force, compared to the case without

Volume 8, Issue 1, 2018 


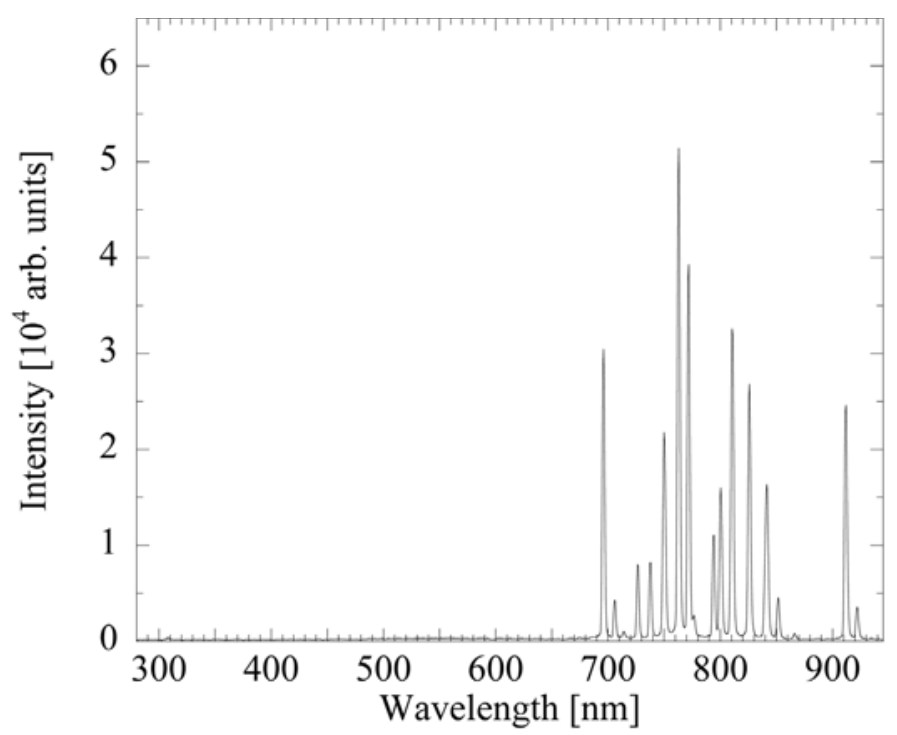

FIG. 2: Emission spectra of argon plasma at $15 \mathrm{~mm}$ from the edge of the powered electrode inside the quartz tube on the downstream side

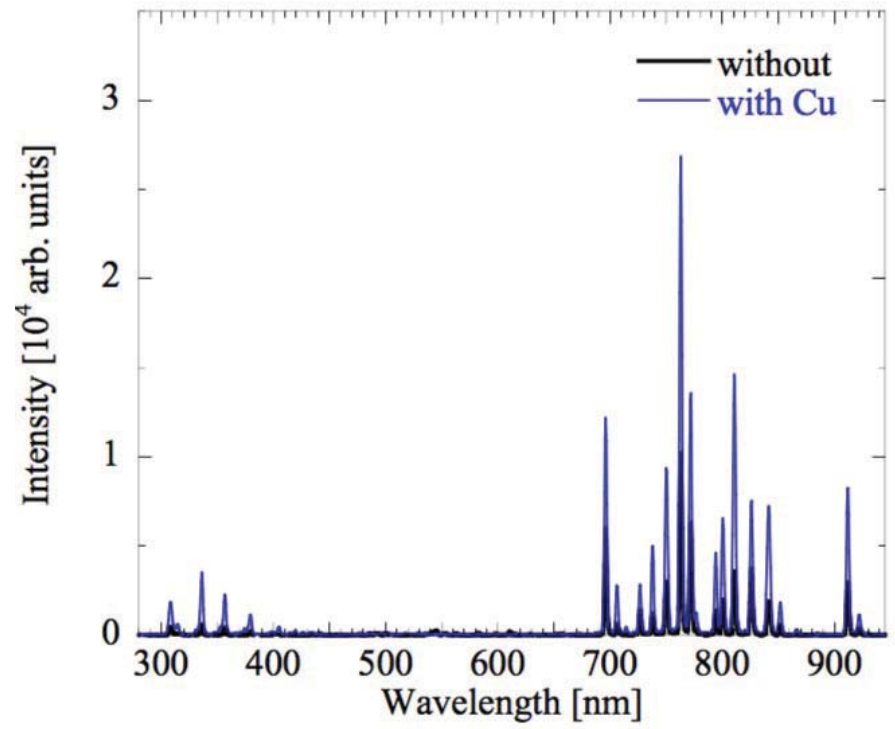

FIG. 3: Emission spectra of argon plasma, oxygen ion, and copper ion at the irradiation point of $10 \mathrm{~mm}$ from the edge of the quartz tube on the downstream side, without and with copper plate, for irradiation by argon plasma 
metal plate. As a result, the plasma is drawn to a metal plate. Because the plasma density increases and the drift velocity of the plasma bullet speeds up, the emission of light strengthens. Figure 4 shows the expanded scale of the spectral lines of argon plasma, oxygen ion, and metal ion, obtained at $10 \mathrm{~mm}$ from the edge of the quartz tube on the downstream side, without and with metal plate. The excitation emission of light was observed in the same wavelength region for each case. Observed wavelengths between 300 and $410 \mathrm{~nm}$ are related to oxygen and metal ions. The centroids of observed wavelength are shown in Tables 1 and 2. Nickel and copper ion excitation appeared by $\mathrm{Ni} \mathrm{I}$ and $\mathrm{Cu}$

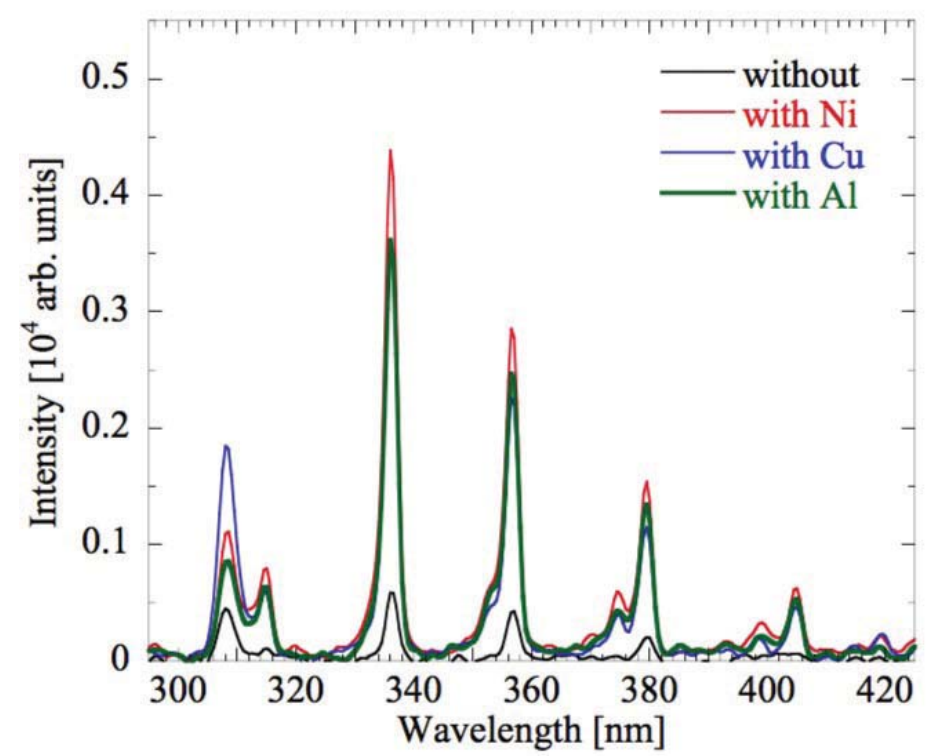

FIG. 4: Expanded scale for emission spectra of the oxygen and metal ions at the irradiation point of $10 \mathrm{~mm}$ from the edge of the quartz tube on the downstream side, without and with metal plate

TABLE 1: Excitation energy and transition strength at the observed wavelengths of argon ion Ar I and oxygen ion O II

\begin{tabular}{lcccc}
\hline Ion & $\begin{array}{c}\text { Excitation energy } \\
\boldsymbol{E}_{\mathbf{n}}(\mathbf{e V})\end{array}$ & $\begin{array}{c}\text { Centroid of observed } \\
\text { wavelength }(\mathbf{n m})\end{array}$ & $\begin{array}{c}\text { Wavelength } \\
\boldsymbol{\lambda}_{\mathbf{n}}(\mathbf{n m})\end{array}$ & $\begin{array}{c}\text { Transition strength } \\
\boldsymbol{g}_{\mathbf{n}} \boldsymbol{A}_{\mathbf{n k}}\left(\mathbf{s}^{-1}\right)\end{array}$ \\
\hline Ar I & 12.907 & 911.67 & 912.29 & $5.67 \times 10^{7}$ \\
Ar I & 13.095 & 800.38 & 801.48 & $4.64 \times 10^{7}$ \\
Ar I & 13.302 & 706.14 & 706.72 & $1.90 \times 10^{7}$ \\
Ar I & 13.302 & 737.84 & 738.39 & $4.24 \times 10^{7}$ \\
O II & 28.67706 & 405.04 & 409.414 & $1.54 \times 10^{7}$ \\
O II & 28.95584 & 336.07 & 337.7146 & $2.66 \times 10^{8}$ \\
O II & 29.58603 & 314.87 & 313.968 & $1.53 \times 10^{8}$ \\
\hline
\end{tabular}

Volume 8, Issue 1, 2018 
TABLE 2: Excitation energy and transition strength at observed wavelengths of nickel ion Ni I, copper ion $\mathrm{Cu}$ I, and aluminum ion $\mathrm{Al}$ II

\begin{tabular}{lcccc}
\hline Ion & $\begin{array}{c}\text { Excitation energy } \\
\boldsymbol{E}_{\mathbf{n}}(\mathbf{e V})\end{array}$ & $\begin{array}{c}\text { Centroid of observed } \\
\text { wavelength }(\mathbf{n m})\end{array}$ & $\begin{array}{c}\text { Wavelength } \\
\boldsymbol{\lambda}_{\mathbf{n}}(\mathbf{n m})\end{array}$ & $\begin{array}{c}\text { Transition strength } \\
\boldsymbol{g}_{\mathbf{n}} \boldsymbol{A}_{\mathbf{n k}}\left(\mathbf{s}^{-1}\right)\end{array}$ \\
\hline $\mathrm{Ni} \mathrm{I}$ & 3.479997 & 356.76 & 357.187 & $3.60 \times 10^{7}$ \\
$\mathrm{Ni} \mathrm{I}$ & 3.705699 & 379.6 & 377.557 & $2.10 \times 10^{7}$ \\
$\mathrm{Ni} \mathrm{I}$ & 4.153573 & 308.23 & 306.462 & $7.70 \times 10^{7}$ \\
$\mathrm{Cu}$ I & 5.10238 & 336.07 & 333.7845 & $3.00 \times 10^{6}$ \\
$\mathrm{Cu}$ I & 5.688311 & 308.23 & 306.3411 & $6.20 \times 10^{6}$ \\
$\mathrm{Cu}$ I & 8.867195 & 405.63 & 406.2641 & $1.26 \times 10^{8}$ \\
$\mathrm{Al} \mathrm{II}$ & 15.30194 & 356.76 & 358.7185 & $5.15 \times 10^{6}$ \\
$\mathrm{Al} \mathrm{II}$ & 16.72787 & 405.04 & 402.65 & $3.48 \times 10^{7}$ \\
$\mathrm{Al} \mathrm{II}$ & 17.26965 & 308.23 & 308.8516 & $5.40 \times 10^{7}$ \\
\hline
\end{tabular}

I. Oxygen ion (O II) excitation appeared at wavelengths of 314, 338, and $409 \mathrm{~nm}$. In these wavelength regions, O I of oxygen ion was not observed. Aluminum ion (Al II) excitation appeared at wavelengths of 309,359 , and $403 \mathrm{~nm}$, because aluminum ion $\mathrm{Al}$ I was not observed except at $\sim 309 \mathrm{~nm}$ in these wavelength regions. Consequently, the wavelength region activated by atmospheric-pressure nonequilibrium argon plasma was limited. We confirmed that each ion corresponding to a wavelength region was activated by argon plasma.

Energy distribution of each ion activated by argon plasma would have MaxwellBoltzmann distribution. The excitation energy $E_{\mathrm{n}}$ and transition strength $g_{\mathrm{n}} A_{\mathrm{nk}}$ on the observed wavelengths of Ar I, O II, Ni I, Cu I, and Al II, taken from the National Institute of Standards and Technology Atomic Spectra Database, ${ }^{28}$ are listed in Tables 1 and 2. The relationship between spectral-line intensity and excitation level was estimated from spectrum line density $I_{\mathrm{n}}$, the wavelength of the $n$ level $1_{\mathrm{n}}, g_{\mathrm{n}} A_{\mathrm{nk}}$, and $E_{\mathrm{n}}$, as follows:

$$
\log \frac{I_{n} \lambda}{g_{n} A_{n k}}=-\frac{5040}{T_{\mathrm{ex}}} E_{n}+\log \frac{N_{0} h c}{U\left(T_{\mathrm{ex}}\right)}
$$

where $T_{\mathrm{ex}}, N_{0}, h, c$, and $U\left(T_{\mathrm{ex}}\right)$ are excitation temperature of the electron, molecular density, Planck's constant, speed of light, and partition function, respectively. ${ }^{27}$ The first term on the right side is related to electron excitation temperature in plasma. The relationships between observed spectral-line intensities and excitation levels for Ar I, without and with metal plate, are shown in Fig. 5. In Figs. 5-7, the average value is estimated from the three shots, error bars denote maximum and minimum values, and the line shows a linear fit. We confirmed that the energy distribution of argon plasma showed Maxwell-Boltzmann distribution in all cases. When observed wavelengths between 300 and $410 \mathrm{~nm}$ were related to oxygen ion, the relationship between observed spectral-line 


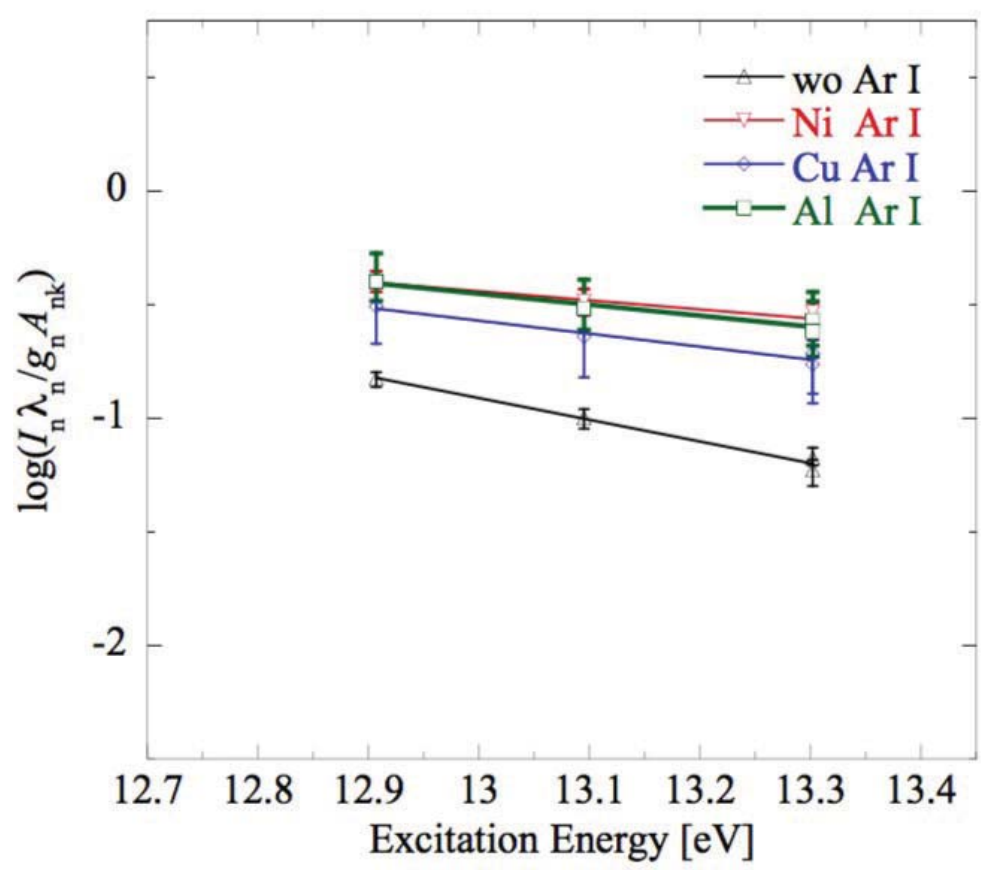

FIG. 5: Relationship between observed spectral-line intensities and excitation levels for argon ion Ar I, without and with metal plate. wo, without.

intensities and excitation levels for O II, without and with metal plate, are shown in Fig. 6. The values of oxygen ion on excitation energy were scattered, compared with those of argon ion. When observed wavelengths between 300 and $410 \mathrm{~nm}$ were related to metal ion, the relationship between observed spectral-line intensities and excitation levels for Ni I, Cu I, and Al II, with metal plate, are shown in Fig. 7. The values of metal ion on excitation energy converged, in comparison to those of oxygen ion. We show the degree of agreement for the fitting line in each distribution in Table 3. The value closer to 1 represents a better match. In the case without metal plate, the ion activated by argon plasma is oxygen ion only. The agreement degree of the oxygen ion is close to 1 . In each case with metal plate, the ions activated by argon plasma are oxygen and metal ions. The agreement degree of metal ion distribution is greater than that of oxygen ion distribution. Oxygen ions as well as metal ions are generated by plasma irradiation. In each observed wavelength, the wavelength due to metal ion activated by argon plasma is dominant, because excitation energy of oxygen ion is greater than that of each metal ion. Consequently, emission of oxygen-ion light would be masked by that of metal ion. Excitation emission of light was observed in the same wavelength region for each case. Consequently, the wavelength region activated by atmospheric-pressure equilibrium argon plasma was limited. In the presence of ion corresponding to wavelength region activated by argon plasma, corresponding ions were activated.

Volume 8, Issue 1, 2018 


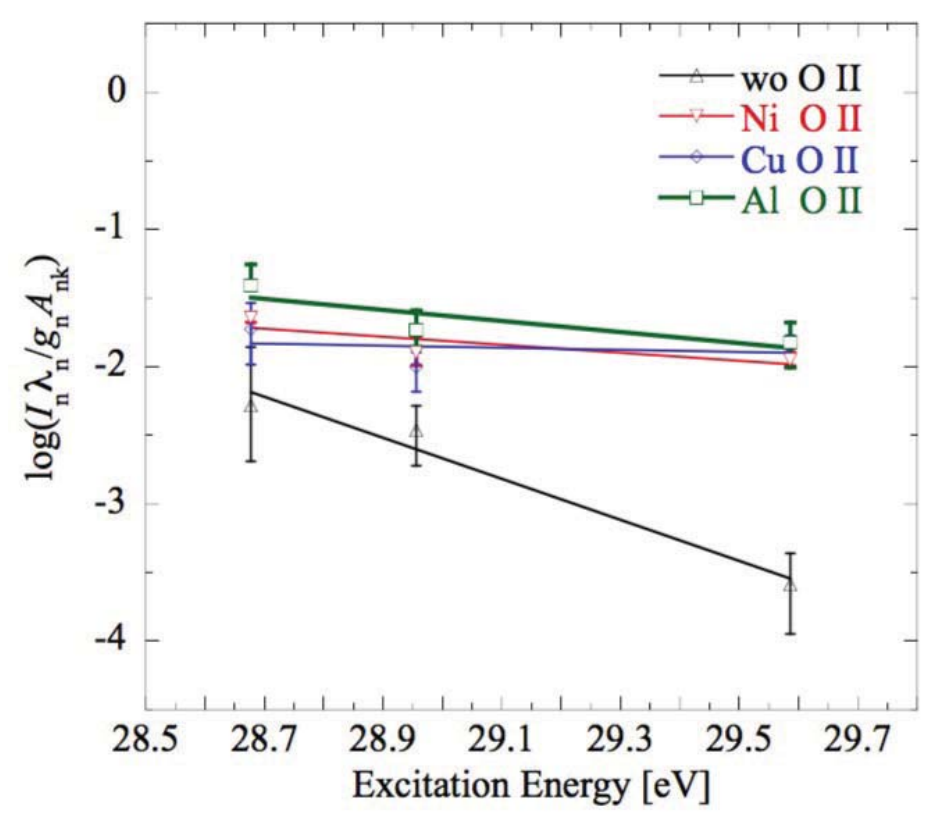

FIG. 6: Relationship between observed spectral-line intensities and excitation levels for oxygen ion O II, without and with metal plate. wo, without.

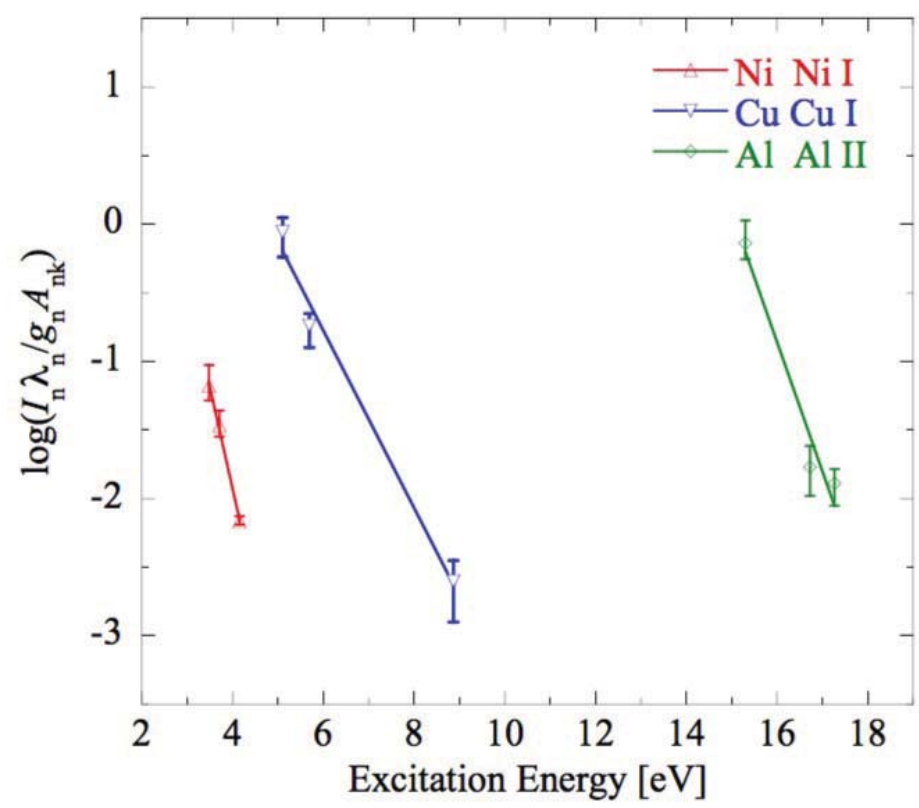

FIG. 7: Relationship between observed spectral-line intensities and excitation levels for metal ions with metal plate 
TABLE 3: Agreement degree of the fitting line on the relationship between observed spectralline intensities and excitation levels for ions

\begin{tabular}{lccc}
\hline Metal plate & Ar I & O II & Metal ion \\
\hline Without & 0.993 & 0.984 & - \\
$\mathrm{Ni}$ & 0.962 & 0.832 & 0.999 \\
$\mathrm{Cu}$ & 0.978 & 0.250 & 0.993 \\
$\mathrm{Al}$ & 0.967 & 0.862 & 0.978 \\
\hline
\end{tabular}

\section{B. Excitation Temperature}

When electron collisions dominate excitation, excitation temperature is closely related to electron temperature. Each excitation temperature shows temperature of plasma due to each activated ion. Metal ions activated by plasma irradiation occur from the surface layer of metal plate. Therefore, excitation temperature for O II infers that the temperature of oxygen plasma activated by argon plasma, and each excitation temperature for metal ion, is approximately equal to the temperature of metal plasma. The temperatures of each plasma are estimated from the relationship between observed spectralline intensities and excitation levels for Ar I, O II, Ni I, Cu I, and Al II; all are listed in Table 4. In the case without metal plate, temperature of argon plasma is higher than that of oxygen plasma. Temperature of argon plasma in all cases is higher than that of plasma activated by argon plasma. In the case with nickel plate, temperature of argon plasma is highest and that of nickel plasma is lowest. Total temperature of plasmas in each case is estimated by adding temperatures of argon plasma and that of plasma activated by argon plasma. In the case without metal plate, total temperature is lowest, because argon plasma does not draw to the wood base. In cases with metal plate, total temperature is almost the same value. Thermal conductivity of each metal is listed in Table 4. Thermal conductivity of $\mathrm{Cu}$ is highest and that of $\mathrm{Ni}$ is lowest. Therefore, the order of temperature of metal plasma accords with order of thermal conductivity. Consequently, thermal energy as the source of total temperature depends on output power from the power supply. Therefore, total thermal energy in all cases is almost the same.

TABLE 4: Plasma temperatures of argon, oxygen, and metal and thermal conductivity of metal

\begin{tabular}{lcccc}
\hline Metal plate & $\begin{array}{c}\text { Argon plasma } \\
\text { temperature (K) }\end{array}$ & $\begin{array}{c}\text { Oxygen or metal } \\
\text { plasma temperature } \\
(\mathbf{K})\end{array}$ & $\begin{array}{c}\text { Total plasma } \\
\text { temperature } \\
(\mathbf{K})\end{array}$ & $\begin{array}{c}\text { Thermal } \\
\text { conductivity } \\
(\mathbf{W} /[\mathbf{m} \text { K] }]) \text { at 298 K }\end{array}$ \\
\hline Without & $5270 \pm 302$ & $3371 \pm 359$ (oxygen) & $8641 \pm 661$ & - \\
$\mathrm{Ni}$ & $12492 \pm 1165$ & $3408 \pm 543$ & $15900 \pm 1708$ & 91 \\
$\mathrm{Cu}$ & $8801 \pm 502$ & $7799 \pm 325$ & $16600 \pm 827$ & 401 \\
$\mathrm{Al}$ & $10427 \pm 1285$ & $5351 \pm 266$ & $15778 \pm 1551$ & 205 \\
\hline
\end{tabular}

Volume 8, Issue 1, 2018 
Heat propagation from argon plasma to metal plate occurs through plasma irradiation. Consequently, heat propagation from argon plasma to copper plate increases compared to cases of other metal plates, because thermal conductivity of $\mathrm{Cu}$ is greatest. Heat loss from argon plasma in the case with copper plate is high, and argon plasma temperature is low. Consequently, in the case of atmospheric-pressure nonequilibrium plasma, plasma is affected by interaction with the object, because energy of the plasma is low, so temperature of nonequilibrium plasma changes by thermal conductivity of an object.

\section{CONCLUSION}

We studied excitation wavelength and temperature in atmospheric-pressure nonequilibrium argon plasma. Excitation wavelengths by plasma irradiation were observed to fall between 300 and $410 \mathrm{~nm}$ outside the quartz tube of the atmosphere region, in all cases with metal plate. Excitation emission of light was observed to occur in the same wavelength region by each case. Observed wavelengths between 300 and $410 \mathrm{~nm}$ are related to oxygen and metal ions. The wavelength region activated by atmosphericpressure nonequilibrium argon plasma was limited. We confirmed that in all cases, energy distribution of argon plasma showed Maxwell-Boltzmann distribution. In the case without metal plate, the ion activated by argon plasma was the oxygen ion only. In each case with metal plate, the ions activated by argon plasma were oxygen and metal ions. Oxygen ions and metal ions are generated by plasma irradiation. In each observed wavelength, the wavelength due to metal ion activated by argon plasma was dominant, because excitation energy of oxygen ion is greater than that of each metal ion. Consequently, emission of light of oxygen ion would be masked by that of metal ion. Excitation emission of light was observed to reside in the same wavelength region with each case. Consequently, the wavelength region activated by atmosphericpressure nonequilibrium argon plasma was limited. In the presence of the ion corresponding to a wavelength region activated by argon plasma, corresponding ions are activated.

In the case without metal plate, temperature of argon plasma is greater than that of oxygen plasma. Temperature of argon plasma in all cases is greater than that of oxygen or metal plasma activated by argon plasma. In the cases with metal plate, total temperature was almost the same value. Thermal conductivity of $\mathrm{Cu}$ is highest and that of $\mathrm{Ni}$ is lowest. Heat propagation from argon plasma to metal plate occurs through plasma irradiation. Consequently, heat propagation from argon plasma to copper plate increases, compared with cases of other metal plates, because thermal conductivity of $\mathrm{Cu}$ is greatest. Heat loss from argon plasma in the case with copper plate is high and temperature of argon plasma is low. Consequently, in the case of atmospheric-pressure nonequilibrium plasma, plasma is affected by the interaction with the object, because energy of the plasma is low, so temperature of nonequilibrium plasma changes by thermal conductivity of the object. 


\section{REFERENCES}

1. Tanaka H, Mizuno M, Ishikawa K, Nakamura K, Kajiyama H, Kano H, Kikkawa F, Hori M. Plasmaactivated medium selectively kills glioblastoma brain tumor cells by down-regulating a survival signaling molecule, AKT kinase. Plasma Med. 2011;1:265-77.

2. Ikehara Y, Sakakita H, Shimizu N, Ikehara S, Nakanishi H. Formation of membrane-like structures in clotted blood by mild plasma treatment during hemostasis. J Photopolym Sci Technol. 2013;26:55-7.

3. Teschke M, Kedzierski J, Finantu-Dinu E, Korzec D, Engemann J. High-speed photographs of a dielectric barrier atmospheric pressure plasma jet. IEEE Trans Plasma Sci. 2005;33:310-1.

4. Walsh J, Kong M. Frequency effects of plasma bullets in atmospheric glow discharges. IEEE Trans Plasma Sci. 2008;36:954-5.

5. Lu X, Naidis G, Laroussi M, Ostrikov K. Guided ionization waves: Theory and experiments. Phys Rep. 2014;540:123-66.

6. Lu X, Naidis G, Laroussi M, Reuter S, Graves D, Ostrikov K. Reactive species in non-equilibrium atmospheric-pressure plasma: Generation, transport, and biological effects. Phys Rep. 2016;630:1-84.

7. Lu X, Laroussi M. Dynamics of an atmospheric pressure plasma plume generated by submicrosecond voltage pulses. J Appl Phys. 2006;100:063302-1-6.

8. Shi J, Zhong F, Zhang J, Liu D, Kong M. A hypersonic plasma bullet train traveling in an atmospheric dielectric-barrier discharge jet. Phys Plasmas. 2008;15:013504-1-5.

9. Sands B, Ganguly B, Tachibana K. Time-resolved imaging of "plasma bullets" in a dielectric capillary atmospheric pressure discharge. IEEE Trans Plasma Sci. 2008;36:956-7.

10. Yambe K, Masuda S. Relation between plasma velocity and power spectrum density in atmosphericpressure plasma plume. Phys Plasmas. 2016;23:093516-1-5.

11. Urabe K, Ito Y, Tachibana K, Ganguly B. Behavior of N2+ ions in He microplasma jet at atmospheric pressure measured by laser induced fluorescence spectroscopy. Appl Phys Express. 2008;1:066004$1-3$.

12. Mericam-Bourdet N, Laroussim M, Begum A, Karakas E. Experimental investigations of plasma bullets. J Phys D Appl Phys. 2009;42:055207-1-7.

13. Karakas E, Koklu M, Laroussi M. Correlation between helium mole fraction and plasma bullet propagation in low temperature plasma jets. J Phys D Appl Phys. 2010;43:0155202-1-5.

14. Yambe K, Furuichi T, Ogura K. Influence of gas flow on plasma length in atmospheric pressure plasma jet. JPS Conf Proc. 2014;1:015084-1-5.

15. Yambe K, Konda K, Ogura K. Influence of flowing helium gas on plasma plume formation in atmospheric pressure plasma. Phys Plasmas. 2015;22:053513-1-6.

16. Yambe K, Saito H. Estimation of flow channel parameters for flowing gas mixed with air in atmospheric-pressure plasma jets. J Phys Soc Jpn. 2017;86:124502-1-5.

17. Yambe K, Sakakita H, Koguchi H, Kiyama S, Ikeda N, Hirano Y. Experimental study on focusing multiple atmospheric-pressure plasma jets. J Plasma Fusion Res. 2009;8:1322-5.

18. Yambe K, Konda K, Ogura K, Sakakita H. Dependence of plasma plume formation on applied voltage waveform in atmospheric-pressure plasma. IEEE Trans Plasma Sci. 2016;44:107-12.

19. Wu S, Xu H, Lu H, Pan Y. Effect of pulse rising time of pulse DC voltage on atmospheric pressure non-equilibrium plasma. Plasma Proc Polym. 2013;10:136-40.

20. Yambe K, Taka S, Ogura K. Relation between plasma plume density and gas flow velocity in atmospheric pressure plasma. Phys Plasmas. 2014;21:043511-1-5.

21. Yambe K, Taka S, Ogura K. Relation between plasma plume charge and length in atmospheric pressure plasma. IEEJ Trans. 2014;9:S13-6.

22. Yambe K, Saito H, Ogura K. Measurement method of plasma current and density in atmospheric pressure plasma jet. IEEJ Trans. 2015;10:614-8.

23. Yambe K, Konda K, Masuda S. Charge dependence of the plasma travel length in atmospheric-pressure plasma. Phys Plasmas. 2016;23:063512-1-7.

Volume 8, Issue 1, 2018 
24. Gessel A, Carbone E, Bruggeman P, Mullen J. Laser scattering on an atmospheric pressure plasma jet: Disentangling Rayleigh, Raman and Thomson scattering. Plasma Sources Sci Technol. 2012;21(1):015003-1-9.

25. Yambe K, Satou S. Investigation of helium plasma temperature in atmospheric-pressure plasma plume using line pair method. Phys Plasmas. 2016;23:023509-1-5.

26. Yambe K, Muraoka S, Nihei T, Abe S. Estimation of excitation temperature by duty ratio of observed period in non-equilibrium plasma. Phys Plasmas. 2017;24:063512-1-8.

27. Miyachi I, Kito Y, Okada K. Distribution of gas temperature and electrode-metal vapor density in an electric power arc determined by the radiated spectral-line intensity. J Inst Electr Eng Jpn. 1967;87;1227-34.

28. Kramida A, Ralchenko Yu, Reader J, NIST ASD Team [database on the Internet]. Gaithersburg (MD): NIST Atomic Spectra Database (ver. 5.3), National Institute of Standards and Technology [cited Sept. 2015]. Available from: http://physics.nist.gov/asd.

29. Engineeringtoolbox.com [homepage on the Internet]. Thermal conductivity of common Materials and Gases [cited Sept. 2015]. Available from: hhttp://www.engineeringtoolbox.com/thermal-conductivityd_429.html. 